\title{
Osteoarthritis is not a disease, but rather an accumulation of predisposing factors. A systematic review
}

\author{
Robert Gherghel ${ }^{1,2, \dagger}$, Daniel-Andrei Iordan ${ }^{3, *,}$, Mircea-Dan Mocanu ${ }^{3, \dagger}$, Ana Onu ${ }^{2, \dagger}$, Ilie Onu ${ }^{1,2, \dagger}$
}

Editor: Mihail HOTETEU, Romanian Association of Balneology, hoteteu@yahoo.com

Reviewers: Constantin Munteanu and Gabriela Dogaru

*Corresponding author: Daniel-Andrei Iordan, „Dunărea de Jos” University, Faculty of Physical Education and Sports, Galați, România, e-mail: daniel.iordan@ugal.ro;

1.Department of Biomedical Sciences, Faculty of Medical Bioengineering, "Grigore T. Popa” University of Medicine and Pharmacy Iasi, România 2.Departments of orthopedics and physiotherapy Micromedica Medical Clinic, Piatra Neamț

3. "Dunărea de Jos" University, Faculty of Physical Education and Sports, Galați, România

$\dagger$ All these authors contributed equally to this paper as senior authors.

\section{Abstract}

Introduction: Although they do not endanger the life of the individual, the major symptoms of osteoarthritis (OA), such as pain, inflammation and dysfunction, it will slowly decrease quality of life and performance, leading finally to disabilities. Due to the fact that this disease has no cure, strategies are still being sought to slow its evolution. The lack of understanding of the predisposing and triggering factors of OA, has led to different approaches to this pathology so discussed, but with modest results. This systematic review aims to debate the main phenomena underlying joint destruction in OA, and etiopathogenic theories.

Materials and Methods: In this study were included 58 bibliographic sources, of which title 39 refers to OA, 6 with inflammation, 28 with cartilage, 3 with chondrocytes, and 5 with synovitis. In this study were discussed the etiopathogenic theories of OA which include: age, alteration of the cartilaginous matrix, alteration of chondrocyte metabolism, microtrauma and major trauma, inflammation of the joints - synovitis and obesity.

Results: Increasing the level of understanding of predisposing factors, the occurrence of acute inflammatory phenomenon and the perpetuation of mechanisms that latently maintain chronic inflammation that over time develops a destructive effect on articular cartilage, would limit the negative effects of OA, delay the evolution and optimally combat that maintain the vicious circle: inflammation $\rightarrow$ production of enzymes $\rightarrow$ chondrolysis $\rightarrow$ inflammation.

Conclusions: These studies contribute significantly to the understanding of destructive phenomena in OA. More studies are needed on the risk factors of $\mathrm{OA}$ and its production mechanisms, to find increasingly effective therapies that limiting its progression.

Keywords: Osteoarthritis, etiopathogenic theories, chronic inflammation, age, obesity, cartilaginous matrixm, chondrocyte metabolism

\section{INTRODUCTION}

Although they do not endanger the life of the individual, the major symptoms of osteoarthritis (OA), such as pain, inflammation, and dysfunction, are treated with great concern by contemporary society because they decrease the quality of life and performance at work leading finally to disability. From physicians to rheumatologists, orthopedists, and physiotherapists, all consider the longterm management of patients with OA a key point, gradually through educating the patient, losing weight, stimulating him to do physical activities, pain management, physiotherapy, intraarticular injections, and finally surgery (1). The lack of an effective strategy for understanding biochemical and biophysical phenomena from the perspective of $\mathrm{OA}$ has led to different approaches to this pathology so discussed, but with modest results. The aim is the most effective therapy and medication to ensure the restoration of joint structures, or at least maintain the integrity of structures and help reduce pain, inflammation, and joint dysfunction syndrome, with few side effects and low long-term costs (2).

\section{Synovial joint}

The joint is that anatomical structure that allows movement in the human body, being made up of elements that connect two or more bones. Thus, in the human body, there are two groups of joints: synovial joints (diarthrosis) in which the participating bones remain separated with the possibility of performing highamplitude movements, and synatrosis in which movement is extremely limited or even zero (3).

To withstand the frictional forces and the distribution of external mechanical loads, the synovial joints have a smooth, resistant, flexible, and slippery specialized viscoelastic tissue of mesenchymal origin, called articular cartilage or hyaline cartilage. It has a unique mechanical behavior but with limited recovery capabilities. Hyaline cartilage is composed of chondrocytes that produce and maintain the extracellular matrix of cartilage. The composition of this tissue includes $65-80 \%$ water, chondrocytes, chondroblasts, type II collagen, and proteoglycans (4). 
Due to the harsh intra-articular mechanical environment, the cartilage must withstand extremely compressive constant loads and effectively absorb mechanical shocks. The behavior of the extracellular matrix is explained, in this sense, by its physico-chemical properties of the movement of water and ions inside the cartilage $(5,6)$. The extracellular matrix consists of proteoglycans and type II collagen, which is hyper hydrated and together with water forms a gel that is in direct contact with the synovial fluid, allowing free exchanges of water. For strength and endurance, the extracellular matrix contains several types of collagen (VI, IX, X, XI), lipids, chondroitin, polysaccharides, hyaluronic acid, hydroxyapatite and a non-collagen protein matrix $(5,6,7)$.

\section{Articular cartilage}

The name Hyaline Cartilage comes from Hyalos [Greek], meaning glass and Cartilago [Latin], meaning cartilage. It is non-vascularized in adult organisms, is resistant, firm, flexible and deforms under mechanical pressure, but recovers after removing the deforming force, thus recovering its original shape. In youngs, it is translucent white-blue and in the elderly, it becomes yellowish and opaque. Macroscopically seen, mature hyaline cartilage has a glassy pearly appearance and is microscopically amorphous, with a high water content, with well-defined gaps incorporating rounded, abundant, inhomogeneous chondrocytes but without blood vessels and nerves. The articular cartilage is avascular, aneural and lymphatic, so its morpho-functional integrity is ensured by feeding with nutrients through a diffusion phenomenon at the level of the synovial covering. In the peripheral regions, trophicity is achieved by the synovial vessels, but its capacity to regenerate after traumatic injuries or wear and tear is considered insignificant by the medical world (8).

Hunter concluded in 1743 that "once is destroyed the articular cartilage, he cannot be repaired," because it is systemically vascular and nervous isolated, so it cannot access the body's normal processes of self-healing and repair available to normal tissues, through inflammatory phenomena. Chondrocytes are surrounded by an extracellular matrix and cannot migrate from one position to another, as in normal tissues (Buckwalter and Mankin, 1998). The impossibility of proliferation from a damaged area to an intact site is doubled by the lack of vascularization and temporary fibrin clots that would temporarily fill the defect, where the chondrocytes could migrate. The integrity of the osteo-articular system conditions the values of flexibility, the mobility of the joints and the extension of the muscular tissue being important factors that influence the amplitude of the movements in all the ontogenetic stages $(9,10)$.

Possible cartilage defects are covered with a fibrous scar tissue generated by mesenchymal chondroprogenetic cells that by division and differentiation will generate counterblast and chondrocyte cartilage cells, along with type I collagen. The small number of newly formed cells leads to cartilage replacement defect with a dense connective tissue, but of poor biomechanical quality that over time will degenerate into OA.

The articular cartilage has a complex anatomical structure formed by four different layers of cells these being divided into areas: superficial, medium, deep and calcified. These layers have different patterns of collagen deposition, chondrocytes and water content. In the upper part of the cartilage we find the superficial layer (tangential area) where the chondrocytes are small and flattened, proteoglycans are found in small numbers, and collagen fibers have a dense arrangement with a uniform diameter and oriented parallel to the surface (11). In the deeper layer (radial area) the chondrocytes are larger, with a more rounded profile, arranged in vertical columns in which the proteoglycan content increases and the collagen fibers acquire an oblique orientation to provide a transition network between the superficial tangential area and the deep radial area (12).

\section{Chondrocytes}

Chondrocytes are mature cells components of cartilaginous tissue, larger than chondroblasts (immature cells), which have undergone a process of synthesis, differentiation and division and are the only cells found in cartilage gaps. They can have a round or oval shape, and through the compression phenomena towards the contact area of the joint they flatten. As a cellular structure, chondrocytes have a round nucleus located close to the periphery of the cell and eosinophilic cytoplasm containing: endoplasmic reticulum, Golgi apparatus, lysosomes, free ribosomes, elongated mitochondria, microtubules, intermediate filaments and actin. Chondrocytes present "reserve substances", these being the glycogen and lipid deposits, used later when needed. They are located in the central area of the cartilage, have a spherical or oval shape with dimensions between 15-40 $\mu$ with a smooth surface and an ovoidshaped hyperchromic nucleus. The type of cartilage is given by the terminal differentiation that determines the character of the cartilage which can be hyaline, fibrous or elastic (13). Chondrocytes produce and maintain the cartilaginous matrix, synthesizing: glycosaminoglycan (GAG), type II collagen and elastic fibers. Along with the synthesis capacity, chondrocytes also produce enzymes that degrade matrix components: collagenase (enzymes that break the peptide bonds in collagen), neutral proteinases and cathepsins $(14,15)$.

The double function of chondrocytes to regulate synthesis but also to degrade cartilage, shows us their agonistantagonist character and the fragile balance in which the joint matrix (ECM) is located. Chondrocytes are also responsible for the process of continuous internal remodeling along with the production of hyaluronic acid with a role in lubricating and keeping the joint surface 
intact. Chondrocytes are present in the human body in a series of anatomical structures and are practically the basic constituent that is part of the three types of cartilage. The tissue arrangement of chondrocytes differs from one anatomical structure to another and is influenced by the presence of gaps and collagen fibers in the matrix $(13,14,15)$.

\section{Chondrolysis}

Chondrolysis is a complex process of progressive destruction of cartilaginous tissue and is found physiologically in the processes of bone formation and remodeling or pathological in the phenomena of degradation of articular cartilage on large bone surfaces in OA. Chondroclasts are the cells involved in the process of chondrolysis but also in the resorption of cartilage.

OA is a complex destructive phenomenon destructive which consist on: chondrolysis, inflammation of the synovium, and marginal osteophytes production. Chondrolysis phenomenon is produced by the chondrocyte which is phenotypically modified on high amounts of metalloprotease enzymes and proinflammatory cytokines such as Interleukin-1 family and casectin (TNF- $\alpha)$. Progressive distruction of articular cartilage is achieved by a damage and abrasion to the support areas, followed by a repair process. The repair process of cartilage is of poor quality, sclerosing the subchondral bone, which will lead to the appearance of cysts and the formation of marginal osteophytes $(14,15)$.

In the 1980s, the medical world and the researchers in this field, considered on the basis of histopathological examinations that OA was a disease only of hyaline cartilage with related bone changes, caused by overload and considerable inflammation of the synovial joint. It is current that $\mathrm{OA}$ is a condition with much more complex implications, and synovitis and the pro-inflammatory mediators that result from it are of overwhelming importance in the pathogenesis of OA with destructive effects on articular cartilage (8).

\section{Osteoarthritis}

OA is not a not a proper disease, but rather a final pathway of the predisposing factors accumulation, such as: age, altered biomechanics, obesity, trauma and microtrauma. How such biochemical and biophysical processes contribute to OA progression is closely related to the interaction of joint lesions, the immune response to joint damage, and the maintenance of chronic inflammation that results in the spread and progression to the clinically recognized OA phenotype. OA has traditionally been classified as a non-inflammatory arthritis and yet the boundary between inflammatory and degenerative arthritis becomes less clear with the recognition of a multitude of ongoing immune processes present in chondrolysis. In the pathogenesis of primary $\mathrm{OA}$, inflammation of the synovial membrane and of the entire joint plays an overwhelming role, along with the presence of synovites $(17,18)$.

From a biochemical point of view, the products of cartilage destruction are released into the synovial fluid and subsequently phagocytosed (the process of incorporation and digestion of bacteria and other foreign bodies in the body) by synovial cells, which leads to synovial over-inflammation. In this process, activated synovial cells in the inflamed synovium, produce catabolic mediators and proinflammators excessive production of proteolytic enzymes, which in turn are responsible for chondrolysis, as part of a repetitive loop phenomenon. The inflammatory response is also amplified by activated synovial B and T cells, along with infiltrated macrophages. To counteract this inflammatory response, the synovium and cartilage will produce antiinflammatory cytokines. The inflamed synovium will also contribute to the formation of osteophytes through BMP (bone morphogenetic proteins). Pathogenic OA is practically a disorder of the balance of synthesis degradation of cartilage and subchondral bone through which cartilaginous lesions, subchondral sclerosis, osteophytosis and bone cysts occur $(17,18,19)$.

Self-healing is limited in OA due to the fact that the joint matrix is not directly supplied with blood, is predominantly aneural, and alymphatic, and specific local healing agents are insufficient. Epidemiologically, age is the first factor - after 55 years the first manifestations of $\mathrm{OA}$ begin as a result of slow chondrolysis; and second factor is sex - in women the higher incidence is in the small joints of the hands, and in men at the hip and knee; the third factor is the race - the Caucasian race having the most to suffer, these being followed by the weight and profession of the individuals (20).

\section{Etiopathogenic theories}

Age

Significant progress has been made in recent years to improve the understanding of how aging contributes to the etiopathogenesis of primary OA. Mitochondrial dysfunction with increasing production of reactive oxygen species (ROS) is closely linked to changes in energy metabolism, with aging affecting the response of chondrocytes to growth factor stimulation. The modified phenotype of chondrocytes in $\mathrm{OA}$ is similar to that of senescence (cell aging) and is manifested by the secretion of increased amounts of chemokines, cytokines and matrix metalloproteinase (MMP) enzymes (21).

Aging of the cartilage is not isolated from the other structures of the joint, it occurs in the context of aging muscles (sarcopenia), bone through increased remodeling and bone loss, fat through increased deposits, the nervous system with changes in proprioception. In this context, the alteration of the joint biomechanics is present both in static and in dynamics, associated with bone deformation, 
with nutrition disorders and chemical composition of the cartilage, all in a regime of chronic local inflammation.

Hyaluronic acid loses its chemical lubricating properties, the molecular weight decreases and by decomposition forms aggregates of proteoglycans that negatively influence the joint by increasing water permeability (22).

\section{Alteration of the cartilaginous matrix}

Multiple factors influence the development and progression of chondrolysis in primary OA. The extracellular matrix (ECM) of the cartilage is actively remodeled by chondrocytes in inflammatory conditions and biomechanical disorders due to wear and constant trauma to the cartilage. This remodeling of the ECM, in turn, modifies the biomechanical environment of chondrocytes, which maintains disease progression in the presence of intra-articular inflammation. Changes in the composition and structure of the ECM prevent the participation of mesenchymal stem cells (MSCs) in the repair process by inhibiting their chondrogenic differentiation. Another change that leads to chondrolysis is the production of point mutations in the complementary DNA which in turn modifies the coding of type II collagen $(23,24,25)$.

In healthy cartilage, chondrocytes maintain a delicate balance between ECM synthesis and degradation and the major components of which we mention type II collagen and aggregate. When this physiological balance fails, the mechanism supporting the ECM loses its physicochemical properties, the matrix begins to decompose due to the oxidative state of the stressed cell environment, and eventually causes apoptosis of chondrocytes. ECM equilibrium failure is due to the high production of ECM degradation enzymes, by inhibiting matrix synthesis with degradation products and pro-inflammatory mediators including cytokines, chemokines, etc. Another feature of the degradation process is the modification of the subchondral bone by osteosclerosis and osteophytosis; along with the degradation of the capsulo-ligamentous apparatus and the weakening of the periatricular muscles $(24,25,26,27,28)$.

Secondary ECM is influenced by rheumatic diseases such as gout, chondrocalcinosis and other genetic diseases such as hemochromatosis (accumulation of iron in the body) and Wilson's disease (accumulation of copper in the body), conditions that lead to changes in the ECM by crystal deposits that stiffen and alter chondrocytes.

Taking into account the non-genetic factors: aging, obesity, along with lifestyle, as the main risk factors for $\mathrm{OA}$, the potential significance of epigenetics in the pathogenesis of this disease is highlighted. Despite extensive research, the pathogenesis and etiology of OA are still unclear. The pathogenesis of OA and implicitly of chondrolysis is given by inflammation and inflammatory mediators both locally and systemically. For rheumatoid arthritis, current therapies are well targeted at mediators that are associated with inflammation and joint destruction and give good results, which is almost impossible to replicate in OA. The mediators responsible for inflammation in $\mathrm{OA}$ are cytokines, chemokines and growth factors, that are found soluble in synovial fluid, which lubricates and maintains joint trophicity $(23,25,29)$.

Adult articular cartilage is a complex tissue as a result of the compilation of matrix proteins, which vary from the superficial to the deep layers and from the loaded areas to the unloaded areas. Cartilage regeneration is inefficient due to the inability of resident chondrocytes to establish a new matrix with the same physicochemical properties as when it was formed during development (29).

\section{Alteration of chondrocyte metabolism}

Joint metabolism is the expression of all biochemical reactions that occur in all structures of joints. Two major processes are present in the metabolism of joint structures: anabolism - through energy consumption and bio-synthesis; catabolism - release of energy and degradation of substances in the joint (30).

Chondrocytes are metabolically active cells that synthesize and regulate the large volume of ECM components such as collagen, glycoproteins, proteoglycans and hyaluronic acid. The metabolic activities of chondrocytes are altered by many factors that are present in their chemical and mechanical environment. The most important of these factors are proinflammatory cytokines and growth factors that have anabolic and catabolic effects. These factors play an important role in the degradation and synthesis of matrix macromolecules. However, the molecular mechanism by growth factors and peptides determine their effects on ECM metabolism has little been studied (31).

Chondrocytes are derived from mesenchymal stem cells and occupy only $1-5 \%$ from the total cartilage tissue, due to the ratio between the volume of the large matrix and that of the cell. The lifespan of codrocytes is regulated by their areas of residence. Because cartilaginous joints are predominantly avascular tissues, chondrocytes rely on the diffusion of nutrients and metabolites from the synovial membrane and subchondral bone. Moreover, these cells operate in a low-oxygen environment with a slow metabolism rate. Due to this, chondrocytes contain low mitochondrial numbers. Chondrocytes are mechanosensitive and participate intensively in the production of ECM, providing the functional and mechanical capacity to withstand the compressive, shear and traction force at the level of diarthrodial joints. The main function of chondrocytes in the superficial (tangential) and middle (radial) area is to synthesize ECM composed of type II, IX and XI collagen and proteoglycans $(32,33,34)$.

Collagen is a macromolecule with the highest rate of spread in the ECM, and represents $60 \%$ of the dry weight 
of the cartilage ensuring mechanical resistance to traction and tissue shearing, while being a good stabilizer of the matrix. Type II collagen represents $90-95 \%$ of the collagen in the ECM and forms fibers and fibers interspersed with proteoglycans as aggregates. Type IX and XI collagens represent $5 \%-10 \%$ of the collagen network of the cartilaginous joint and provide support for cross-linked fibrillar collagen. Deep-area chondrocytes are terminologically differentiated and actively synthesized by type X collagen (34).

Proteoglycans represent the second group of macromolecules and are glycosylated proteins that are present in almost all extracellular matrices of connective tissues. The major biological function of proteoglycans derives from the physicochemical characteristics of the glycosaminoglycan molecule, which ensures water retention in the tissue, allowing it to withstand compressive forces. The most resistant and abundant proteoglycan in cartilage is agrecan. These proteoglycans have distinct biological functions, in addition to their hydrodynamic functions, and their involvement in many aspects of cellular and tissue activities has been demonstrated. Specific interactions between proteoglycans (via glycosaminoglycan and basic protein components) and macromolecules in the extracellular matrix are key factors in the functions of proteoglycans $(36,37,38)$.

\section{Microtraumas and major traumas}

Some studies show that joint injuries substantially increase the risk of OA, which continues to increase with the patient's age at the time of trauma, and others have reported that it ranges from 20 to $50 \%$ in patients who have had trauma. They represent about $12 \%$ of all OA cases. Common causes leading to OA include intraarticular fractures and meniscal, ligament and cartilage injuries (39).

Joint injuries, such as sprains or dislocations, often lead to a progressive process of severe debilitating condition known as acute post-traumatic arthritis (PTA). It can occur at any age, in any joint and can develop from any kind of acute physical trauma. Although a single trauma can sometimes be enough to induce arthropathy, it is known that repeated injuries and excess body weight increase the risk of PTA. Acute symptoms that result from joint trauma include swelling, synovial effusions, severe pain, and sometimes internal bleeding. Usually, recovery occurs spontaneously in 2-3 months. The persistence of symptoms after this period should deserve attention and only after 6 months, in clinical practice, it can be considered pathological and so-called acutechronic traumatic arthritis. Thus, chronic PTA can be an inflammatory condition that persists over time, after a common trauma. The most common chronic PTA is posttraumatic OA $(39,40)$.
The ankle is the most affected joint, accounting for 54$78 \%$ of over 300,000 injuries occurring in the United States alone each year. However, epidemiologically related knee injuries remain the most prevalent worldwide, with 700,000 cases annually in the US and $12.5 \%$ of post-traumatic OA. At the end of the ranking, the shoulder, hip and fist remain.

A major disadvantage is that post-traumatic OA can only be diagnosed clinically at the onset of the symptomatic phase, which can be highly variable. Posttraumatic OA can occur earlier than less than a year, or remain asymptomatic for a long time, even 10-20 years after trauma. Currents of opinion in the world of science are for both variants of post-traumatic $\mathrm{OA}$ and it is increasingly believed that the development of $\mathrm{OA}$ in injured joints begins during the initial traumatic event through intra-articular pathogenic processes, such as apoptosis of joint chondrocytes. , remodeling of the subchondral bone, cellular infiltration and release of inflammatory mediators in the synovial fluid $(40,41)$.

The pathogenesis of chronic PTA is not fully understood and a variety of factors may be involved, including genetic predisposition, epigenetic changes, mechanobiological and inflammatory mechanisms. Despite the increased knowledge in recent years of the potential pathogenic mechanisms that occur in damaged joints, specific predictive markers remain incomplete. Current treatments for joint injuries fail to prevent the evolution of PTA in OA $(41,42)$.

OA can also be caused by recurrent dislocations, reduced fractures - incorrectly consolidated or osteonecrosis phenomena, which are based on severe trauma. Microfractures cause cartilage remodeling by calcification, altering the subchondral bone by increasing its hardness and reducing the height of the cartilage in uncalcified areas, making it extremely vulnerable to mechanical overload. However, the degradation by metabolic and degenerative alterations of the cartilage will appear at the immobilized joints for a long time and the atrophy of the articular cartilage is observed in this case. PTA treatment is a difficult problem to manage. Currently, biochemical markers that predict or correlate disease progression are completely absent, and therapeutic interventions are limited to immobilization and stabilization of the joint. Anti-inflammatory therapy, especially intra-articular infiltrations, inhibits cytokine production and can provide an effective approach to reducing or preventing chronic disability $(43,44,45)$.

\section{Joint inflammation - Synovitis}

OA was considered a "wear and tear" disease until the 2000s, which eventually led to the loss of articular cartilage. The technological leap in molecular biology in the 1990s has profoundly changed this paradigm. The discovery that several soluble mediators, such as 
cytokines or prostaglandins, can increase the production of MMPs by chondrocytes, led to the first steps in the formation of an "inflammatory" theory. However, it took another decade for synovitis to be accepted as a critical feature of OA, and some studies claim that it could be the "engine" of OA (46). Recent experimental data have shown that subchondral bone may play a substantial role in chondrolysis processes, acting as a mechanical shock absorber, but also a source of inflammatory mediators involved in the pain process of OA and in the degradation of the deep layer of articular cartilage. OA not only affect cartilage, it konwn that is more just an complex disease, with inflammatory mediators released by cartilage, bone and synovium. Low-grade inflammation induced by metabolic syndrome, immunity and inflammation, are some of the most recent arguments in favor of the inflammatory theory of OA (17).

Synovitis is a local inflammation characterized by swelling of the joints - an important element in the clinical picture of OA, being attributed to inflammation and confirming the presence of synovitis due to thickening of the synovium or effusion. When patients suffer from OA outbreaks (night pain, morning stiffness) they usually have parallel joint effusions, as seen in classic inflammatory arthropathies, such as rheumatoid arthritis $(46,47,48)$ (table 1$)$.

High sensitivity systemic levels to protein $\mathrm{C}$, reflect synovial inflammation in patients with $\mathrm{OA}$ and are associated with high levels of pain. What is certain is that synovial inflammation that occurs in meniscal trauma is associated with increased pain and knee dysfunction. The most accepted hypothesis is that once it degrades, the cartilage fragments enter the joint and connect to the synovium. These being interpreted as "foreign bodies", synovial cells react by producing inflammatory mediators, found in synovial fluid. These mediators can activate the chondrocytes present in the superficial layer of the cartilage, which leads to the synthesis of MMP and finally, to the increase of the cartilage degradation. Mediators can also induce synovial angiogenesis and increase the synthesis of inflammatory cytokines and MMPs by synovial cells (vicious circle). Thus, synovitis behaves as a trigger of $\mathrm{OA}$ and perpetuates chondrolysis $(49,50,51)$.

Another recent theory involves synovial tissue as the main trigger of the OA process. Inflammation will initiate and maintain pathological changes in the articular cartilage through increased release of enzymes and / or leukocytes in the joint space such as serum proteases, cathepsin-D and elastase. In the process of inflammation in OA, inflammatory mediators stimulate chondrocytes to produce lysis enzymes, maintaining a vicious cycle consisting of (52): inflammation $\rightarrow$ enzymes $\rightarrow$ chondrolysis $\rightarrow$ inflammation
Table 1. Reference level of acute joint inflammation, responsible for early degradation of articular cartilage, as a perspective for the evaluation of synovitis

\begin{tabular}{|c|c|}
\hline $\begin{array}{l}\text { Reference level } \\
\text { of joint } \\
\text { inflammation } \\
\end{array}$ & Observations \\
\hline Clinical & $\begin{array}{l}\text { Effusion, swelling of the joints, } \\
\text { Palpable synovitis, } \\
\text { Sudden increase pain, night pain and } \\
\text { morning stiffness. }\end{array}$ \\
\hline histologically & $\begin{array}{l}\text { Synovial hypertrophy and hyperplasia, } \\
\text { Infiltration of mononuclear cells (monocytes } \\
\text { macrophages, activated B cells and } \mathrm{T} \\
\text { cells), } \\
\text { Macrophages block and form giant } \\
\text { multinucleated cells for enhanced } \\
\text { phagocytosis, } \\
\text { Increased angiogenesis, } \\
\text { Synovitis at the edge of degenerative } \\
\text { cartilage. }\end{array}$ \\
\hline Macromolecular & $\begin{array}{l}\text { Production and / or release of } \\
\text { proinflammatory cytokines (TNF, IL-1p, IL- } \\
6 \text {, IL-8, IL-15, IL-17, IL-18, IL-21), } \\
\text { Increased production of protaglandins and } \\
\text { nitric oxide, } \\
\text { Increasing the activity of adhesion } \\
\text { molecules (ICAM-1, VCAM-1) in the } \\
\text { synovial, } \\
\text { Increasing the activity of matrix } \\
\text { metalloproteases (MMP-1, MMP-3, MMP- } \\
9 \text {, MMP-13) and ADAMTS, } \\
\text { Production of adipokines (leptin, } \\
\text { adiponectin) } \\
\text { Involvement of macrophages in the } \\
\text { formation of osteophytes by BMP, } \\
\text { Insufficient release of anti-inflammatory } \\
\text { cytokines (IL-4, IL-10, IL-13, IL-1Ra), } \\
\text { Release of pro-inflammatory } \\
\text { neurotransmitters and pain neurotransmitters }\end{array}$ \\
\hline $\begin{array}{l}\text { Medical } \\
\text { imaging }\end{array}$ & $\begin{array}{l}\text { Synovial hypertrophy revealed by MRI } \\
\text { examination, } \\
\text { Musculoskeletal ultrasound provides } \\
\text { information on synovial effusion and } \\
\text { hypertrophy, } \\
\text { The association of MRI examinations, } \\
\text { ultrasound and synovitis clinic can predict } \\
\text { the progression and degradation of articular } \\
\text { cartilage } \\
\text { Without x-Ray association. }\end{array}$ \\
\hline $\begin{array}{l}\text { Treatment / } \\
\text { interventions }\end{array}$ & $\begin{array}{l}\text { Intra-articular infiltrations based on } \\
\text { corticosteroids may have a positive short- } \\
\text { term effect on clinical symptoms and } \\
\text { synovial hypertrophy, } \\
\text { Biological response } \\
\text { (immunotherapy) have potential effects on } \\
\text { affected structures. }\end{array}$ \\
\hline
\end{tabular}




\section{Obesity}

Obesity has always been associated with OA, especially of the knee and hip, with a general negative impact on the musculoskeletal system. Obesity leads to static and dynamic disorders, excessive joint loading and alteration of biomechanical patterns, along with hormonal disorders and cytokines. Obesity is associated with the incidence and progression of $\mathrm{OA}$ in both weight-bearing and weightless joints, with a high rate of chondrolysis progression with a disabling end. Weight loss in OA can bring clinically significant improvements in pain and delay the progression of structural joint damage (53).

Some authors have suggested that OA is not just a disease of aging or metabolic stress of the joints, but rather a metabolic disorder in which various interconnected glue, metabolic and humoral mediators contribute to the initiation and progression of the disease process. Obesity clearly leads to an increase in the load on the load-bearing joint, which may be the most important mechanical contribution to the chondrolysis phenomenon. The pathophysiology of obesity in OA is multifactorial. Joint injuries are caused by mechanical factors, of which we mention: abnormal intrarticular forces, decreased general muscle tone and disturbances of biomechanics during locomotion. In obese metabolic factors, the risk of OA increases, especially in the loadbearing and non-load-bearing joints. Obesity is characterized by a low inflammatory condition and is addressed to several systems and organs. The pathological mechanisms are not fully understood but it is known that altered adipokine expression will lead to the destruction and remodeling of joint tissues $(53,54,55)$. The adipokines from which we list leptin and adiponectin exert their influence on joint tissue, including articular cartilage, synovial and subchondral bone. Leptin and adiponectin are the most widespread adipokines in the intra-articular environment, and their receptors are expressed on the surface of chondrocytes, synoviocytes and osteoblasts $(53,56)$.

Leptin increases the levels of degrading enzymes: nitric oxide, matrix metalloproteinases (MMPs), and the production of proinflammatory cytokines. Adipokine levels in obese people can be particularly important, as obesity can produce a biochemical environment in which chondrocytes develop abnormal behavior under stress. The role of adiponectin in joint disease is not well known nor its pro-inflammatory and anti-inflammatory properties compared to its systemic anti-inflammatory effects. Leptin and adiponectin levels are significantly elevated in people with OA. A recent study on significant weight loss in obese subjects with knee OA showed decreased circulating leptin levels and increased circulating levels of adiponectin $(57,58)$.

\section{For the future}

The studies undertaken will make an original and significant contribution to the in-depth knowledge of chondrolysis phenomena in OA, and the effectiveness of limiting progression through the targeted use of combined therapies at different stages and needs. More studies are needed on the risk factors of OA and its production mechanisms. Decreased consumption of analgesics and systemic anti-inflammatory drugs prescribed by doctors with harmful effects on the digestive system, excretory system and cardiovascular system is absolutely necessary in medical practice. OA is a progressive, disabling condition with few effective treatment strategies. Increasing the level of understanding of predisposing factors, the occurrence of acute inflammatory phenomenon and the perpetuation of mechanisms that latently maintain chronic inflammation that over time develops a destructive effect on articular cartilage, would limit the negative effects of OA, delay the evolution and optimally combat that maintain the vicious circle: inflammation $\rightarrow$ production of enzymes with lysis effect $\rightarrow$ chondrolysis $\rightarrow$ inflammation.

\section{Declaration of conflict of interests:}

There is no conflict of interest for any of the authors regarding this paper.

Acknowledgments: The work of Daniel Andrei IORDAN was supported by the project "ANTREPRENOR-DOC", Contract no. 36355/23.05.2019, financed by The Human Capital Operational Program2014e2020 (POCU), Romania.

\section{References:}

1. Giuseppe Musumeci, Flavia Concetta Aiello, Marta Anna Szychlinska, Michelino Di Rosa, Paola Castrogiovanni, and Ali Mobasheri. Osteoarthritis in the XXIst Century: Risk Factors and Behaviours that Influence Disease Onset and Progression. Int J Mol Sci. 2015 Mar; 16(3): 6093-6112. Published online 2015 Mar 16. doi: 10.3390/ijms16036093

2. Roland W Moskowitz. The burden of osteoarthritis: clinical and quality-of-life issues. Am J Manag Care. 2009 Sep;15(8 Suppl):S223-9

3. Ifrim M. Iliescu A. Anatomia și biomecanica educaţiei fizice și sportului, Editura didactică și pedagogică (1978), pag 103-104

4. Muir H. The chondrocyte, architect of cartilage. Biomechanics, structure, function and molecular biology of cartilage matrix macromolecules. Bioessays. (1995)(12):1039-48.

5. Mostakhdemin M, Nand A, Ramezani M. Articular and Artificial Cartilage, Characteristics, Properties and Testing Approaches-A Review. Polymers (Basel). 2021 Jun 18;13(12):2000. doi: 10.3390/polym13122000. PMID: 34207194; PMCID: PMC8234542.

6. Patel JM, Wise BC, Bonnevie ED, Mauck RL. A Systematic Review and Guide to Mechanical Testing for Articular Cartilage Tissue Engineering. Tissue Eng Part C 
Methods. $2019 \quad$ Oct;25(10):593-608. doi: 10.1089/ten.TEC.2019.0116. Epub 2019 Sep 30. PMID: 31288616 ; PMCID: PMC6791482.

7. Luo Y, Sinkeviciute D, He Y, Karsdal M, Henrotin Y, Mobasheri A, Önnerfjord P, Bay-Jensen A. The minor collagens in articular cartilage. Protein Cell. 2017 Aug;8(8):560-572. doi: 10.1007/s13238-017-0377-7. Epub 2017 Feb 17. PMID: 28213717 ; PMCID: PMC5546929.

8. Mocanu GD. Kinesiologie [Internet]. Galati University Press; 2016 [cited 2020 Dec 10]. Available from: http://arthra.ugal.ro/handle/123456789/6286

9. Mocanu GD. Study on Educating the Flexibility of the Lower Body Muscles of the Students From the Specialized Faculties Through the Curricular Practical Activities. GYMNASIUM. 2020 Jun 29;XXI(1):15-33.

10. Francois $\mathrm{Ng}$, kee Kwong, Myron Spector. Principles of Regenerative Medicine (2008), Pages 766-781

11. Alan Ivkovic, Damir Hudetz and Marko Pecina. ARTICULAR CARTILAGE REPAIR IN ATHLETES, Current Concepts and Future Treatment, (2015) Aspetar Sports Medicine Juournal page 257,

12. Mainil-Varlet $P$, et al. International Cartilage Repair Society. Histological assessment of cartilage repair: a report by the Histology Endpoint Committee of the International Cartilage Repair Society (ICRS). J Bone Joint Surg Am. 2003; 85-A Suppl 2:45-57. PMID: 12721345.

13. Phull AR, Eo SH, Abbas Q, Ahmed M, Kim SJ. Applications of Chondrocyte-Based Cartilage Engineering: An Overview. Biomed Res Int. 2016;2016:1879837. doi: 10.1155/2016/1879837. Epub 2016 Aug 18. PMID: 27631002 ; PMCID: PMC5007317.

14. Charlier E, Relic B, Deroyer C, Malaise O, Neuville S, Collée J, Malaise MG, De Seny D. Insights on Molecular Mechanisms of Chondrocytes Death in Osteoarthritis. Int $\mathbf{J}$ Mol Sci. 2016 Dec 20;17(12):2146. doi: 10.3390/ijms17122146. PMID: 27999417; PMCID: PMC5187946.

15. Kozhemyakina E, Lassar AB, Zelzer E. A pathway to bone: signaling molecules and transcription factors involved in chondrocyte development and maturation. Development. 2015 Mar 1;142(5):817-31. doi: 10.1242/dev.105536. PMID: 25715393; PMCID: PMC4352987.

16. Hall AC. The Role of Chondrocyte Morphology and Volume in Controlling Phenotype-Implications for Osteoarthritis, Cartilage Repair, and Cartilage Engineering. Curr Rheumatol Rep. 2019 Jun 15;21(8):38. doi: 10.1007/s11926-019-0837-6. PMID: 31203465; PMCID: PMC6571082.

17. Berenbaum F. Osteoarthritis as an inflammatory disease (osteoarthritis is notosteoarthrosis!). Osteoarthritis Cartilage 2013; 21:16-21.

18. Jeremy Sokolove and Christin M. Lepus, Role of inflammation in the pathogenesis of osteoarthritis: latest findings and interpretations Ther Adv Musculoskelet Dis. 2013 Apr; 5(2): 77-94.

19. Alexander Mathiessen and Philip G. Conaghan. Synovitis in osteoarthritis: current understanding with therapeutic implications. Arthritis Res Ther. 2017; 19: 18

20. Sun AR, Udduttula A, Li J, Liu Y, Ren PG, Zhang P. Cartilage tissue engineering for obesity-induced osteoarthritis: Physiology, challenges, and future prospects.
J Orthop Translat. 2020 Sep 28;26:3-15. doi: 10.1016/j.jot.2020.07.004. PMID: 33437618; PMCID: PMC7773977.

21. Richard F. Loeser. Aging and Osteoarthritis. Curr Opin Rheumatol. Author manuscript; available in PMC 2012 Sep 1. Published in final edited form as: Curr Opin Rheumatol. 2011 Sep; 23(5): 492-496.

22. Ghosh P, Guidolin D. Potential mechanism of action of intra-articular hyaluronan therapy in osteoarthritis: are the effects molecular weight dependent? Semin Arthritis Rheum. 2002 Aug; 32(1):10-37.

23. Maricela Maldonado and Jin Nam. The Role of Changes in Extracellular Matrix of Cartilage in the Presence of Inflammation on the Pathology of Osteoarthritis, BioMed Research International Volume 2013, Article ID 284873

24. Akkiraju H, Nohe A. Role of Chondrocytes in Cartilage Formation, Progression of Osteoarthritis and Cartilage Regeneration. J Dev Biol. 2015 Dec;3(4):177-192. doi: 10.3390/jdb3040177. Epub 2015 Dec 18. PMID: 27347486; PMCID: PMC4916494.

25. Maldonado M, Nam J. The role of changes in extracellular matrix of cartilage in the presence of inflammation on the pathology of osteoarthritis. Biomed Res Int. 2013;2013:284873. doi: 10.1155/2013/284873. Epub 2013 Aug 28. PMID: 24069595; PMCID: PMC3771246.

26. Yoshimura H, Muneta T, Nimura A, Yokoyama A, Koga H, Sekiya I. Comparison of rat mesenchymal stem cells derived from bone marrow, synovium, periosteum, adipose tissue, and muscle. Cell Tissue Res. 2007;327:449-462.

27. Tuan RS. Cellular signaling in developmental chondrogenesis: N-cadherin, Wnts, and BMP-2. J. Bone Joint Surg. Am. 2003;85-A(Suppl. 2):137-141

28. Blaney Davidson EN, Vitters EL, van Lent PL, van de Loo FA, van den Berg WB, van der Kraan PM. Elevated extracellular matrix production and degradation upon bone morphogenetic protein-2 (BMP-2) stimulation point toward a role for BMP-2 in cartilage repair and remodeling. Arthritis Res. Ther. 2007;9:R102.

29. Michiel Beekhuizen. Inflammatory mediators in osteoarthritis - Identification and role in cartilage repair, 2014, ISBN/EAN 978-90-9028322-7

30. Bora FW Jr, Miller G. Joint physiology, cartilage metabolism, and the etiology of osteoarthritis. Hand Clin. 1987 Aug; 3(3):325-36. PMID: 3308909.

31. Hemanth Akkiraju and Anja Nohe. Role of Chondrocytes in Cartilage Formation, Progression of Osteoarthritis and Cartilage Regeneration. Journal of Developmental Biology ISSN 2221-3759,2015, 3, 177-192;

32. Solchaga LA, Penick KJ, Welter JF. Chondrogenic differentiation of bone marrow-derived mesenchymal stem cells: tips and tricks. Methods Mol Biol. 2011;698:253-78. doi: 10.1007/978-1-60761-999-4_20. PMID: 21431525; PMCID: PMC3106977.

33. Alexis L. et al. Comparison of the Chondrogenic Differentiation Potential of Equine Synovial MembraneDerived and Bone Marrow-Derived Mesenchymal Stem Cells. Front. Vet. Sci., 06 June 2019

34. Sanchez-Adams J, Leddy HA, McNulty AL, O'Conor CJ, Guilak F. The mechanobiology of articular cartilage: bearing the burden of osteoarthritis. Curr Rheumatol Rep. 
2014 Oct;16(10):451. doi: 10.1007/s11926-014-0451-6. PMID: 25182679; PMCID: PMC4682660.

35. N.S.Gudmann M.A.Karsdal. Biochemistry of Collagens, Laminins and Elastin Structure, Function and Biomarkers. Chapter 2 - Type II Collagen 2016, Pages 13-20

36. Yanagishita M.. Function of proteoglycans in the extracellular matrix. Acta Pathol Jpn. 1993 Jun; 43(6):28393.

37. Yanagishita M. Function of proteoglycans in the extracellular matrix. Acta Pathol Jpn. 1993 Jun;43(6):28393. doi: 10.1111/j.1440-1827.1993.tb02569.x. PMID: 8346704.

38. B. Henderson, J.C.W. Edwards and E.R. Pettipher. Mechanisms and Models in Rheumatoid Arthritis. Academic Press. 1995 Elsevier .ISBN978-0-12-340440-4

39. Jiménez G, Cobo-Molinos J, Antich C, López-Ruiz E. Osteoarthritis: Trauma vs Disease. Adv Exp Med Biol. 2018;1059:63-83. doi: 10.1007/978-3-319-76735-2_3. PMID: 29736569.

40. Punzi L, Galozzi P, Luisetto R, Favero M, Ramonda R, Oliviero F, Scanu A. Post-traumatic arthritis: overview on pathogenic mechanisms and role of inflammation. RMD Open. 2016 Sep 6;2(2):e000279. doi: 10.1136/rmdopen2016-000279. PMID: 27651925; PMCID: PMC5013366.

41. J.Lieberthal, N.Sambamurthy, C.R.Scanzello. Inflammation in joint injury and post-traumatic osteoarthritis. Osteoarthritis and Cartilage. Volume 23, Issue 11, November 2015, Pages 1825-1834

42. S.R. Goldring. Pathophysiology of post-traumatic arthritis. Osteoarthritis and Cartilage. Volume 20, SUPPLEMENT 1, S7, April 01, 2012

43. Salaffi F. Ciapetti A, Carotti M,The sources of pain in osteoarthritis: a pathophysiological review.Reumatismo. 2014 Jun 6;66(1):57-7

44. Fujiwara S, Hiranaka T, Hida Y, Matsuda S, Uemoto H. Osteoarthritis with permanent dislocation of the patella treated by total knee arthroplasty through a lateral approach: A report of two cases. Int J Surg Case Rep. 2017;38:73-77. doi: 10.1016/j.ijscr.2017.07.012. Epub 2017 Jul 8. PMID: 28743096; PMCID: PMC5524422.

45. Mäenpää H, Lehto MU. Patellofemoral osteoarthritis after patellar dislocation. Clin Orthop Relat Res. 1997 Jun;(339):156-62. doi: 10.1097/00003086-19970600000021. PMID: 9186214.

46. Mathiessen A, Conaghan PG. Synovitis in osteoarthritis: current understanding with therapeutic implications. Arthritis Res Ther. 2017 Feb 2;19(1):18. doi: 10.1186/s13075-017-1229-9. PMID: 28148295; PMCID: PMC5289060.

47. Ishibashi K, Sasaki E, Ota S, Chiba D, Yamamoto Y, Tsuda E, Yoshikuni S, Ihara K, Ishibashi Y. Detection of synovitis in early knee osteoarthritis by MRI and serum biomarkers in Japanese general population. Sci Rep. 2020 Jul 23;10(1):12310. doi: 10.1038/s41598-020-69328-w. PMID: 32704147; PMCID: PMC7378056.

48. Van Ginckel A, Wittoek R, De Mits S, Calders P. Repetitive Knee Bending and Synovitis in Individuals at Risk of and With Knee Osteoarthritis: Data From the Foundation for the National Institutes of Health Osteoarthritis Biomarkers Consortium. Arthritis Care Res (Hoboken). 2019 Oct;71(10):1372-1378. doi: 10.1002/acr.23760. PMID: 30221482; PMCID: PMC6421112.

49. Smith JW, Martins TB, Gopez E, Johnson T, Hill HR, Rosenberg TD. Significance of C-reactive protein in osteoarthritis and total knee arthroplasty outcomes. Ther Adv Musculoskelet Dis. 2012 Oct;4(5):315-25. doi: 10.1177/1759720X12455959. PMID: 23024709; PMCID: PMC3458617.

50. Babaei M, Javadian Y, Narimani H, Ranaei M, Heidari B, Basereh H, Gholinia H, Firouzjahi A. Correlation between systemic markers of inflammation and local synovitis in knee osteoarthritis. Caspian J Intern Med. 2019 Fall;10(4):383-387. doi: 10.22088/cjim.10.4.383. PMID: 31814935; PMCID: PMC6856926.

51. D. Pearle et al. Elevated high-sensitivity C-reactive protein levels are associated with local inflammatory findings in patients with osteoarthritis. OsteoArthritis and Cartilage (2007) $15,516 \mathrm{e} 523$

52. Man GS, Mologhianu G. Osteoarthritis pathogenesis - a complex process that involves the entire joint. J Med Life. 2014 Mar 15;7(1):37-41. Epub 2014 Mar 25. PMID: 24653755; PMCID: PMC3956093.

53. King LK, March L, Anandacoomarasamy A. Obesity \& osteoarthritis. Indian J Med Res. 2013 ;138(2):185-93. PMID: 24056594; PMCID: PMC3788203.

54. Reyes C, Leyland KM, Peat G, Cooper C, Arden NK, Prieto-Alhambra D. Association Between Overweight and Obesity and Risk of Clinically Diagnosed Knee, Hip, and Hand Osteoarthritis: A Population-Based Cohort Study. Arthritis Rheumatol. 2016 Aug;68(8):1869-75. doi: 10.1002/art.39707. PMID: 27059260; PMCID: PMC4966641.

55. Bray GA, Heisel WE, Afshin A, Jensen MD, Dietz WH, Long M, Kushner RF, Daniels SR, Wadden TA, Tsai AG, $\mathrm{Hu}$ FB, Jakicic JM, Ryan DH, Wolfe BM, Inge TH. The Science of Obesity Management: An Endocrine Society Scientific Statement. Endocr Rev. 2018 Apr 1;39(2):79132. doi: 10.1210/er.2017-00253. PMID: 29518206; PMCID: PMC5888222.

56. Azamar-Llamas D, Hernández-Molina G, Ramos-Ávalos B, Furuzawa-Carballeda J. Adipokine Contribution to the Pathogenesis of Osteoarthritis. Mediators Inflamm. 2017;2017:5468023. doi: 10.1155/2017/5468023. Epub 2017 Apr 8. PMID: 28490838; PMCID: PMC5401756.

57. Cordero-Barreal A, González-Rodríguez M, RuizFernández C, Eldjoudi DA, AbdElHafez YRF, Lago F, Conde J, Gómez R, González-Gay MA, Mobasheri A, Pino J, Gualillo O. An Update on the Role of Leptin in the Immuno-Metabolism of Cartilage. Int J Mol Sci. 2021 Feb 27;22(5):2411. doi: 10.3390/ijms22052411. PMID: 33673730; PMCID: PMC7957536.

58. Yan M, Zhang J, Yang H, Sun Y. The role of leptin in osteoarthritis. Medicine (Baltimore). 2018 Apr;97(14):e0257. doi: 10.1097/MD.0000000000010257. PMID: 29620639; PMCID: PMC5902277. 